Fundación

Miguel Lillo

Tucumán

Argentina

\title{
Apuntes sobre el muestrario, colección de referencia y base de datos digital para estudios arqueológicos vinculados al contenido vegetal del patrimonio culinario de los Andes Centro-Sur, América del Sur
}

\author{
Notes on the collection of samples, the reference collection and \\ the database of archaeological studies related to the use of edible \\ plants in the culinary heritage of the South-Central Andes, South \\ America
}

Musaubach, M. Gabriela*1,2,3 (1); Osvaldo Ahumada 3,4 (1); Anabela Plos 5,6,7 (1); Héctor Sato ${ }^{3,4}{ }^{(1)}$; H. Priscila Romero ${ }^{1}{ }^{10}$; L. Tatiana Albornoz ${ }^{1} \mathbb{D}^{\circ}$;

G. Luciana Sejas Paliari ${ }^{10}$; Pilar Babot 8,9 (D)

1 Facultad de Humanidades y Ciencias Sociales, Universidad Nacional de Jujuy (FHyCS, UNJu); Otero 262, (4600) San Salvador de Jujuy, Jujuy, Argentina.

2 Instituto de Datación y Arqueometría, Universidad Nacional de Jujuy (InDyA, UNJu/CONICET/UNT/ Gob. de Jujuy), Av. Gral. Martijena S/N, 4612, Palpalá, Jujuy, Argentina.

3 Instituto de Ecorregiones Andinas, Consejo Nacional de Investigaciones Científicas y Técnicas (INECOA, CONICET - UNJu) Av. Bolivia 1239, (4600) San Salvador de Jujuy, Argentina.

${ }^{4}$ Cátedra de Botánica General - Herbario (JUA). Facultad de Ciencias Agrarias, UNJu. Alberdi 47, CP4600, San Salvador de Jujuy, Jujuy, Argentina.

5 Museo Argentino de Ciencias Naturales, Consejo Nacional de Investigaciones Científicas y Técnicas (MACN-CONICET), Av. Ángel Gallardo 470, (C1405DJR) Ciudad Autónoma de Buenos Aires, Argentina.

6 GBIF Argentina, Av. Ángel Gallardo 470, (C1405DJR) Ciudad Autónoma de Buenos Aires, Argentina.

7 Sistema Nacional de Datos Biológicos, Ministerio de Ciencia, Tecnología e Innovación Productiva (SNDB-MinCyT), Godoy Cruz 2320, (C1425FQD) Ciudad Autónoma de Buenos Aires, Argentina.

8 Instituto de Arqueología y Museo, Facultad de Ciencias Naturales e Instituto Miguel Lillo, Universidad Nacional de Tucumán (IAM, FCN e IML, UNT), San Martín 1545, (4000) San Miguel de Tucumán, Tucumán, Argentina.

9 Instituto Superior de Estudios Sociales, Consejo Nacional de Investigaciones Científicas y Técnicas (ISES, CONICET/UNT), Córdoba 191, (4000) San Miguel de Tucumán, Tucumán, Argentina.

* Autor Corresponsal: gmusaubach@fhycs.unju.edu.ar

\footnotetext{
Ref. bibliográfica: Musaubach, M. G.; Ahumada, O.; Plos, A.; Sato, H.; Romero, H. P.; Albornoz, L. T.; Sejas Paliari, G. L.; Babot, P. 2021. Apuntes sobre el muestrario, colección de referencia y base de datos digital para estudios arqueológicos vinculados al contenido vegetal del patrimonio culinario de los Andes CentroSur, América del Sur. Lilloa 58 (2): 115-130. doi: https://doi.org/10.30550/j.lil/2021.58.2/2021.11.03

- Recibido: 30 de septiembre 2021 - Aceptado: 3 de noviembre 2021 - Publicado en línea: 11 de noviembre 2021.

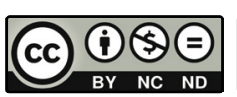

open 2 access

> URL de la revista: http://lilloa.lillo.org.ar

- Esta obra está bajo una Licencia Creative Commons Atribución - No

Comercial - Sin Obra Derivada 4.0 Internacional.
} 


\title{
RESUMEN
}

En el marco de la tendencia global hacia la accesibilidad de las bases de datos, se reseñan los protocolos diseñados para la confección del muestrario y la colección histológica utilizados en el estudio arqueobotánico del patrimonio culinario CentroSurandino, así como su sistematización y puesta a disposición digital. Las muestras de cultivos andinos son productos provenientes de la agricultura familiar a pequeña escala en el sector centro y centro-sur de la Quebrada de Humahuaca. Además, incluyen productos procesados elaborados a partir de estos cultivos (harinas, semillas tostadas, mote, entre otros), los cuales fueron obtenidos en ferias y mercados de la región. Finalmente, se incorporan también las muestras obtenidas durante las experiencias etnoarqueológicas y etnobotánicas de campo con las comunidades locales. Al presente la colección comprende plantas andinas y diversos productos de su elaboración culinaria tales como chicha de maíz y de maní, varios tipos de harinas de maíz (Zea mays L.) -capia, puimada, tostada, para api-, diferentes taxa de semillas como Phaseolus vulgaris L., Chenopodium quinoa Wild., Amarantáceas y marlos con cariopses de las variedades de Zea mays L. que se cultivan tradicionalmente en Jujuy. La colección está pensada como un espacio abierto a nuevas incorporaciones en la medida en que progresan los trabajos de campo y experimentales. Estas muestras se analizaron y registraron con los protocolos de descripción e identificación utilizados en las investigaciones arqueobotánicas. El conocimiento y las prácticas culinarias relacionadas con el procesamiento, el servicio de alimentos y el consumo de taxones andinos comestibles se recuperan y sistematizan en una base de datos digital. Además de la utilidad de este material de referencia para el estudio arqueobotánico, su descripción y registro permite no sólo su catalogación en el patrimonio del Herbario JUA, sino que también aporta a la valorización del patrimonio biocultural comunitario. Así, mediante esta colección, se contribuye a la valorización, visibilización, salvaguardia y gestión del patrimonio culinario quebradeño.

Palabras clave - Arqueobotánica; bases de datos; cultivos andinos; fitolitos; granos de almidón.

\begin{abstract}
Within the framework of the global trend towards database accessibility, we review the designed protocols for the preparation of the Andean crops collection of samples and histological collection used in the archaeobotanical study of SouthCentral Andes culinary heritage as well as their systematization and digitalWithin the framework of the global trend towards database accessibility, we review the designed protocols for the preparation of the Andean crops collection of samples and histological collection used in the archaeobotanical study of South-Central Andes culinary heritage as well as their systematization and digital availability. Samples of Andean crops are small-scale family farming products from the central and south central sector of the Quebrada de Humahuaca. Besides, both collections include processed products made from these crops which were collected from fairs and markets. Finally, samples obtained during our ethnoarchaeological and ethnobotanical field-
\end{abstract}


work, are also incorporated. At present, the collection includes Andean crops and several culinary products such as corn and peanuts chicha, different types of corn flour (Zea mays L.) -capia, puimada, toasted, for api-, different types of seeds such as Phaseolus vulgaris L., Chenopodium quinoa Wild., Amaranthaceae and cobs with caryopses of Zea mays L. varieties traditionally cultivated in Jujuy. The collection has been designed as a space open to new incorporations as field and experimental work progress. The samples were analyzed and described following the description and identification protocols used in our archaeobotanical investigations. Culinary knowledge and practices related to processing, food serving and consumption of edible Andean taxa are retrieved and systematized in a digital database. The reference materials, their description and record are not only relevant for archaeobotanical studies, but they also contribute to the valorisation of the community bio-cultural heritage, allowing us not only to catalogue it in the JUA Herbarium heritage, but also to contribute to the enhancement, visibility, safeguarding and management of South-Central Andes culinary heritage.

Keywords - Archaeobotany; database; Andean crops; phytoliths; starch grains.

\section{INTRODUCCIÓN}

Las colecciones de historia natural son la mayor fuente de datos sobre biodiversidad fuera de la naturaleza misma. En conjunto, las colecciones del mundo incluyen alrededor de tres mil millones de especímenes y documentan más de 300 años de exploración humana activa y los restos fósiles brindan vislumbres adicionales de la profunda historia biológica del planeta. Dichas colecciones y materiales auxiliares, incluidas imágenes, notas de coleccionistas, secuencias de genes (ADN-ARN), mediciones, etc., contienen cantidades colosales de datos que deben digitalizarse y compartirse. Sin embargo, se estima que sólo el 10\% de las colecciones del mundo se han digitalizado y solo una parte de ellas son de acceso público a través de Internet (Woelfle et al., 2011).

Hoy en día, digitalizar una colección (o sea, pasar de un formato analógico a uno digital) no es el final del camino. El movimiento de Ciencia Abierta (Woelfle et al., 2011) procura que los resultados de investigaciones científicas y sus datos sean accesibles a todos los niveles de una sociedad, sean investigadores, aficionados o profesionales. Es mediante portales, donde es posible abrir los resultados de las investigaciones.

Los portales, para ofrecer datos abiertos, deben cumplir una serie de requisitos que hoy se resumen bajo los principios FAIR (Encontrables, Accesibles, Interoperables y Reutilizables, por su sigla en inglés Findable, Accessible, Interoperable, Reusable) (Wilkinson et al., 2016). Estos principios indican que para que un dato sea considerado abierto debe ser: hallable, accesible, interoperable y reutilizable.

Los estudios arqueobotánicos, interesados en las interacciones entre los humanos y las plantas, no escapan a estos requerimientos y a la vez, se benefician de ellos (Lodwick, 2019; Kansa et al. 2020; Karoune, 2020). Esto queda demostrado, por 
ejemplo, en el apoyo y difusión que brinda la International Phytolith Society (IPS) a las colecciones de referencia digitales sobre fitolitos, así como al aval institucional que brinda a iniciativas que buscan la socialización de los datos (ver por ejemplo https://phytoliths.org/ips-standing-committees/). Es desde esta perspectiva que se encuentran en investigación los saberes y prácticas culinarias de las sociedades prehispánicas asociados a los cultivos andinos del pasado de la Quebrada de Humahuaca (Jujuy, Argentina). Esta iniciativa surgió al considerar que si bien existen numerosos estudios etnobotánicos y referidos a botánica aplicada en esa área (Vignale y Gurni, 2001; Lambaré et al., 2015; Villalba, 2019, entre otros), los saberes de las poblaciones prehispánicas referidos a la flora útil para la alimentación habían sido poco trabajados (Albeck, 1992/1993; Pazzarelli, 2008; Nielsen, 2010; Ortiz y Heit, 2012; Pazzarelli y Lema, 2018). De este modo, la investigación en la que se encuadra este trabajo se focaliza en estudios arqueobotánicos de base microscópica en los residuos vegetales de procesamiento culinario y consumo retenidos en el instrumental arqueológico. Dichos estudios permiten precisar los usos del equipamiento culinario prehispánico y su rol en los procesos alimenticios antiguos (ver por ejemplo Musaubach et al., 2019; Musaubach y Scaro, 2019; López Geronazzo et al., 2019; Musaubach y Scaro, 2020a, 2020b).

Las investigaciones que se presentan se enmarcan en el interés de estudiar el patrimonio culinario prehispánico Surandino y sus prácticas sociales asociadas desde una perspectiva arqueobotánica, iniciando esta indagación en la Quebrada de $\mathrm{Hu}-$ mahuaca como caso de estudio. Este conocimiento sobre la culinaria prehispánica se considera como la raíz ancestral del patrimonio cultural gastronómico de dicha quebrada.

Una de las principales metas del proyecto es generar una Base de Datos de Saberes y Prácticas Culinarias Quebradeñas que incluya recetas tradicionales, así como el relevamiento de los cultivos andinos utilizados como ingredientes de dichas preparaciones culinarias. Acompañan a esta base de datos digital, tres elementos de suma relevancia para las investigaciones: el herbario, el muestrario y la histoteca con preparados de granos de almidón y fitolitos de sílice y calcio.

El objetivo de este trabajo es presentar el esquema elaborado para la sistematización y socialización de la información culinaria prehispánica y reseñar los protocolos diseñados para la confección del muestrario, histoteca y base de datos de granos de almidón y fitolitos de sílice y calcio de cultivos andinos que forman parte de las investigaciones arqueobotánicas en desarrollo sobre este patrimonio CentroSurandino. Estos tres cuerpos de materiales de referencia (Fig. 1) se complementan mutuamente y se estructuran siguiendo los criterios adecuados para los estudios arqueobotánicos. 


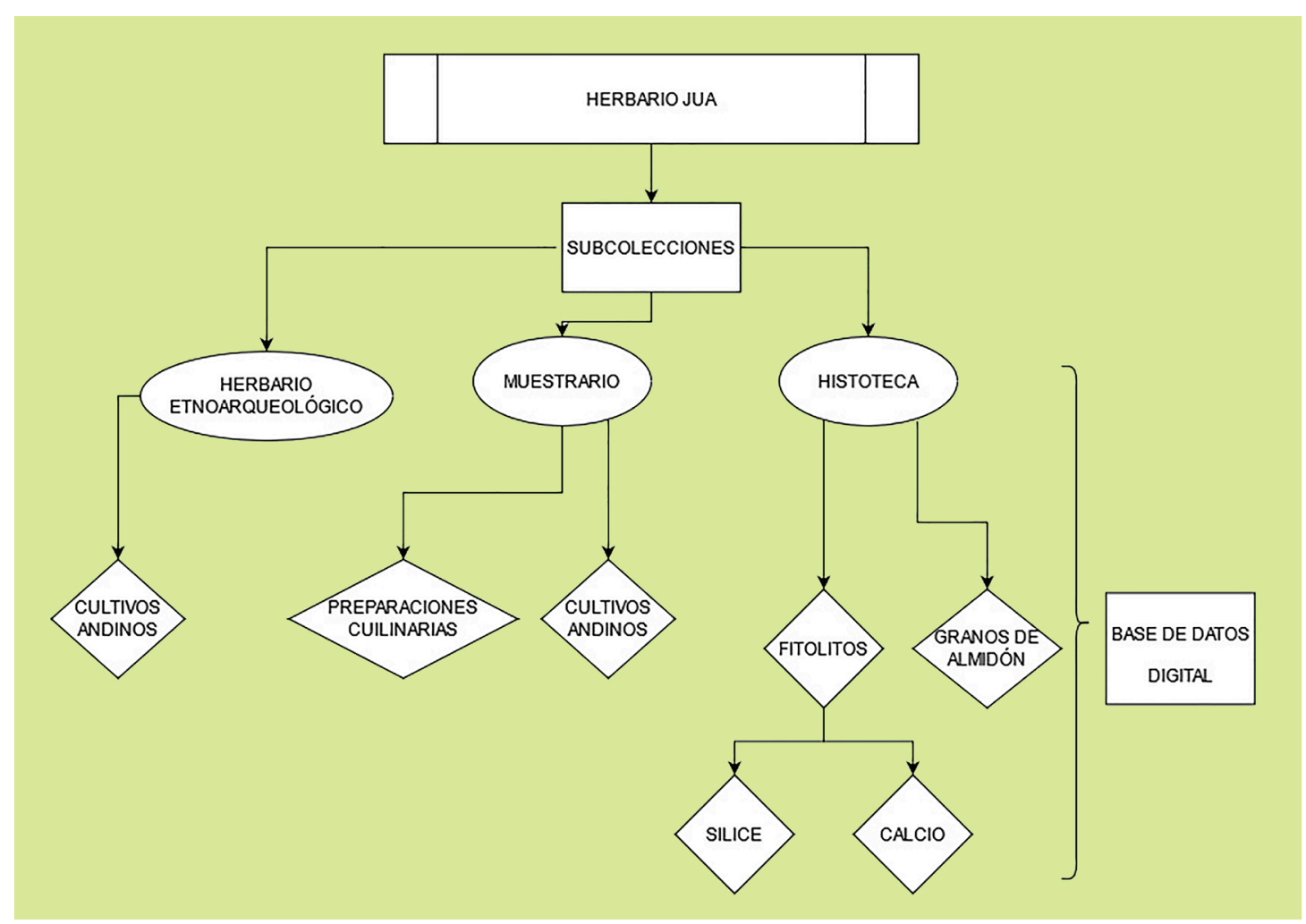

Fig. 1. Esquema con el detalle de los tres tipos de subcolecciones y la base de datos digital.

Fig. 1. Scheme with the three types of sub collections and the digital database.

\section{PROPUESTA METODOLÓGICA}

\section{1) Construcción, descripción y generación de bases de datos}

Un herbario es una colección de plantas prensadas secas y conservadas entre papeles, guardadas en muebles especiales, para que mantengan, de la mejor manera, la forma y la posición de los órganos vegetativos y reproductivos como en el estado viviente (Katinas, 2001). El herbario JUA (significa Jujuy-Agronomía) está ubicado en la Facultad de Ciencias Agrarias (UNJu), y fue elaborado por los integrantes de la Cátedra de Botánica General, desde abril de 1981. El mismo está inscripto en el Index Herbariourum, y cuenta con 15.000 ejemplares conservados y guardados en catorce (14) armarios metálicos guarda-herbarios. JUA pertenece a la Red Nacional de Colecciones Biológicas (RNCB).

Por su parte, un muestrario es una colección de plantas o fragmentos de plantas (semillas, frutos, fibras, tallos, raíces, entre otros) en estado natural o transformadas por procedimientos tecnológicos de diversa índole como prácticas culinarias o aquellos vinculados con la confección de productos artesanales (Arenas y Martínez, 2012). Al igual que un herbario, el muestrario debe ser accesible para consulta y corroboración y la información etnobotánica registrada en relación a la muestra se consigna en una etiqueta bien visible. Cada muestra y material testigo se etiqueta tomando en cuenta la información obtenida en campo; nombre, número de inventario, nombre científico y común de la planta, localidad de colecta, hábitat, caracteres 
de la planta, información sobre usos y tipos de procesamientos culinarios (recetas), datos del colector/a y la fecha de recolección. Todo el material se organiza bajo asignaturas específicas, por ejemplo, siguiendo un criterio de uso. Los muestrarios son un tipo de colección de gran utilidad para el trabajo etnobotánico y arqueobotánico que reúne especímenes que suelen o pueden no estar incorporados en un herbario estándar y que, además, se acompañan de la información referente a los usos dados a las plantas por la sociedad (Arenas y Martínez, 2012).

El muestrario de cultivos andinos y preparaciones culinarias del Herbario JUA está compuesto tanto por plantas en estado natural o seco, como transformadas mediante el procesamiento culinario. En la Fig. 2 se ilustran las diferentes etapas del registro y procesamiento de muestras de algunas de las hierbas medicinales/aromáticas de la Puna y la Quebrada de Humahuaca colectadas, así como de algunas razas de maíces y sus preparaciones culinarias tradicionales (chicha, mote, tijtincha) colectadas durante el trabajo de campo.

Las etapas del protocolo seguido para el registro y catalogación de los ejemplares de la colección incluyen: Fig. 2A) la obtención de ejemplares en mercados, ferias
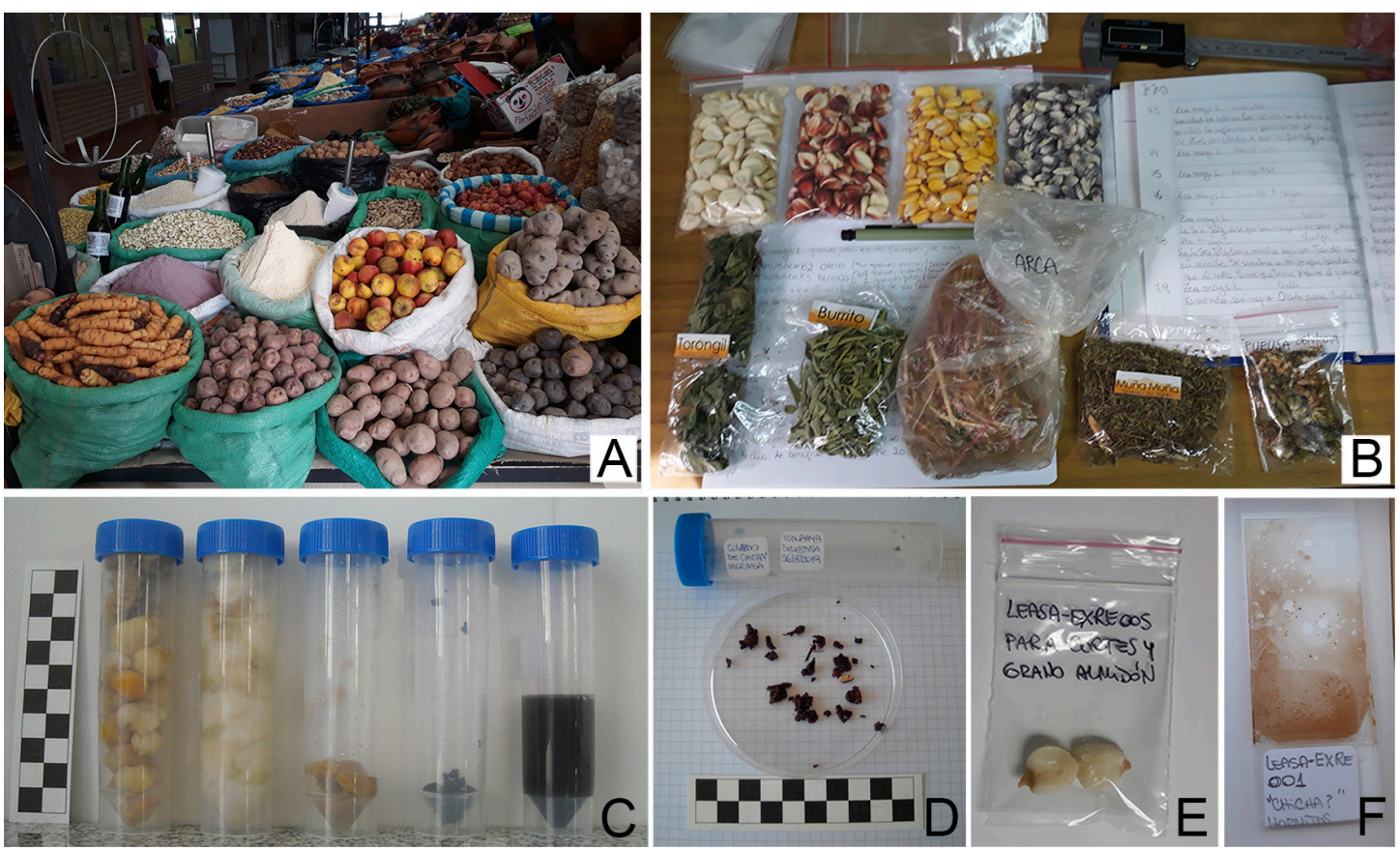

Fig. 2. Etapas del protocolo propuesto para el registro y catalogación de los ejemplares de la colección. A) Obtención de ejemplares en el Mercado de La Quiaca, Jujuy, Argentina. B) Catalogación e inventario del material en estado seco en el gabinete del Herbario JUA. C) Catalogación e inventario de las preparaciones culinarias en el gabinete arqueobotánico. D) Descripción macroscópica de las preparaciones culinarias. E) Bodegaje de material en estado fresco/húmedo. F) Montaje de las muestras de la colección histológica.

Fig. 2. Protocol stages for the registration and cataloguing of the collected specimens. A) Acquisition of specimens in the La Quiaca Market, Jujuy, Argentina. B) Cataloguing and inventory of the dry material in the JUA Herbarium lab. C) Cataloguing and inventory of culinary preparations in the archaeobotanical cabinet. D) Macroscopic description of culinary preparations. E) Storage of material in fresh/humid state. F) Assembly of the histological collection samples. 
tradicionales y trabajos de campo, 2B) la catalogación e inventario del material en estado seco en el herbario, 2C) la catalogación e inventario de las preparaciones culinarias que integrarán el muestrario en el gabinete arqueobotánico, 2D) la descripción macroscópica de las preparaciones culinarias, $2 \mathrm{E}$ ) el bodegaje de material en estado fresco/húmedo y $2 \mathrm{~F}$ ) el muestreo, montaje, análisis, descripción y almacenaje de las muestras de la colección histológica.

1.1. Construcción.-Los ejemplares son obtenidos en colectas etnoarqueológicas en terreno, a partir de trabajos de campo realizados en chacras de pequeños productores de la zona. Otros ejemplares son adquiridos en locales comerciales, festivales y ferias de temáticas vinculadas a temas gastronómicos y agrícolas, tales como las realizadas en las localidades de San Salvador de Jujuy, Tumbaya, Tumbaya Grande, Humahuaca, La Quiaca, Tilcara, El Perchel y Volcán. También se incorporan muestras de preparaciones culinarias obtenidas durante las experiencias etnoarqueológicas y experimentales con las comunidades locales en la quebrada.

Los saberes culinarios se recuperan mediante tres vías complementarias. En el caso de las entrevistas semiestructuradas realizadas durante las experiencias en el campo, las mismas se enmarcan en los acuerdos mediados por el consentimiento libre e informado que se realiza durante la vinculación de los miembros del proyecto con los y las entrevistados/as. En este caso, los entrevistados son consultados si es o no de su preferencia el hecho de que sus recetas sean de acceso público. Otras fuentes de información son los recetarios tradicionales publicados, así como videos documentales que se encuentran en las redes sociales de las y los cocineros tradicionales.

Mediante la elaboración de preparados histológicos y calcinaciones en seco del material (Piperno y Pearsall, 1998) se obtienen los preparados de fitolitos y granos de almidón presentes en los taxa culinarios que forman parte de la colección de referencia de cultivos andinos. Algunos de los taxa culinarios que han sido procesados hasta el momento incluyen las razas de maíces (Zea mays L.) que se cultivan en la Quebrada, los porotos de colores (Phaseolus vulgaris L.), o las semillas de cereales andinos como la quínoa (Chenopodium quinoa Wild). Ambos, cultivados en Tumbaya Grande. Dichos preparados son realizados para los distintos órganos de las plantas, es decir flor, brácteas, inflorescencias, semillas, frutos, tubérculos o raíces modificadas. Este procedimiento se lleva a cabo para todos los especímenes de plantas, fragmentos de plantas o preparaciones culinarias que conforman el herbario y muestrario.

Los preparados de la histoteca se confeccionan según técnicas convencionales de anatomía vegetal, tanto para los análisis histológicos como microhistoquímicos (Johansen, 1940; Zarlavsky, 2014). Entre las primeras se pueden mencionar: lavado, hidratación (dependiendo de las características de la muestra y del tipo de reactivo a utilizar) y ablandamiento; cortes a mano alzada o con micrótomo (según características del material) y raspados histológicos y técnicas de diafanización adaptadas para los estudios arqueobotánicos. La principal técnica de reacción microhistoquímica usada para detectar granos de almidón es la tinción con lugol (Dickau, 2005; Peto et al., 2013; Raviele, 2011; Crowther, 2012; Babot et al., 2014). 
1.2. Metodología para la descripción de las muestras histológicas. - Para todas las muestras histológicas obtenidas, se montan dos preparados (duplicados) de cada muestra. Uno, semipermanente, es utilizado para realizar las descripciones de los fitolitos y granos de almidón. Este tipo de preparados (semipermanentes) son los que usualmente se utilizan en las investigaciones arqueobotánicas para poder realizar las descripciones 3D de los microindicadores botánicos. Como medio de montaje se utilizan una o dos gotas de agua con glicerina o aceite de inmersión sobre cada portaobjeto ya que ambos presentan índices de refracción adecuados para el contraste de almidones y fitolitos. Luego, se realiza otro preparado definitivo montado en Bálsamo de Canadá sintético, con similares propiedades ópticas que los anteriores.

Para describir y clasificar los contenidos en cada preparado se utiliza la microscopia de luz transmitida con polarización. En este trabajo se usó un microscopio Trinocular "Arcano" XSZ-107BN, con set de polarización incorporado. Las mediciones y micrografías se realizaron con una cámara de video "Arcano" KPA 5.0 megapíxeles.

Para describir los granos de almidón y fitolitos se siguen los protocolos de identificación y caracterización utilizados en los estudios arqueobotánicos (Korstanje y Babot, 2007; Musaubach et al., 2013; Babot et al., 2017; Musaubach y Babot, 2019). Para su descripción y clasificación se adaptaron los descriptores y atributos propuestos en el International Code for Phytolith Nomenclature 2.0-ICPN 2.0 (International Committee for Phytolith Taxonomy [ICPT], 2019), el International Code for Starch Nomenclature ([ICSN] 2011), Babot (2007) y Pagan Jiménez (2015). Siguiendo a Babot et al. (2014), Babot y Musaubach (2019) y el ICSN (2011), se realizó la descripción de los granos de almidón y los fitolitos que presentan daños y modificaciones (cambios morfológicos y ópticos) producto de los procesos físico-químicos ocurridos durante las técnicas de transformaciones culinarias de los alimentos. En la Fig. 3 se ejemplifican micrografías de la colección de referencia de fitolitos y granos de almidón que forman parte de la colección. Fig. 3 A y B) se ejemplifica el registro morfométrico de los granos de almidón de Zea mays L. en una muestra de "harina para $a p i$ ", con luz normal y con luz polarizada, $3 \mathrm{C}$ y D) registro de los daños tafonómicos producidos por las prácticas culinarias, con luz normal y polarizada, $3 \mathrm{E}$ ) morfotipos de fitolitos articulados de calcinados de Poaceae.

1.3. Generación de bases de datos. - La información obtenida se consigna en la base de datos digital sistematizando aquella referida a los cultivos andinos y usos alimenticios. A continuación, se detallan los principales descriptores y atributos de la base de datos, agrupados en tres bloques.

1.3.1. Bloque I. Diversidad biológica en Darwin Core.- El material a partir del cual se realizan los preparados de referencia, es incluido en la base de datos y al mismo se refieren los granos de almidón y fitolitos observados posteriormente. De estos materiales, que se encuentran depositados en el Herbario JUA, se relevaron los campos indicados para un registro biológico (https://dwc.tdwg.org/terms/), mediante 

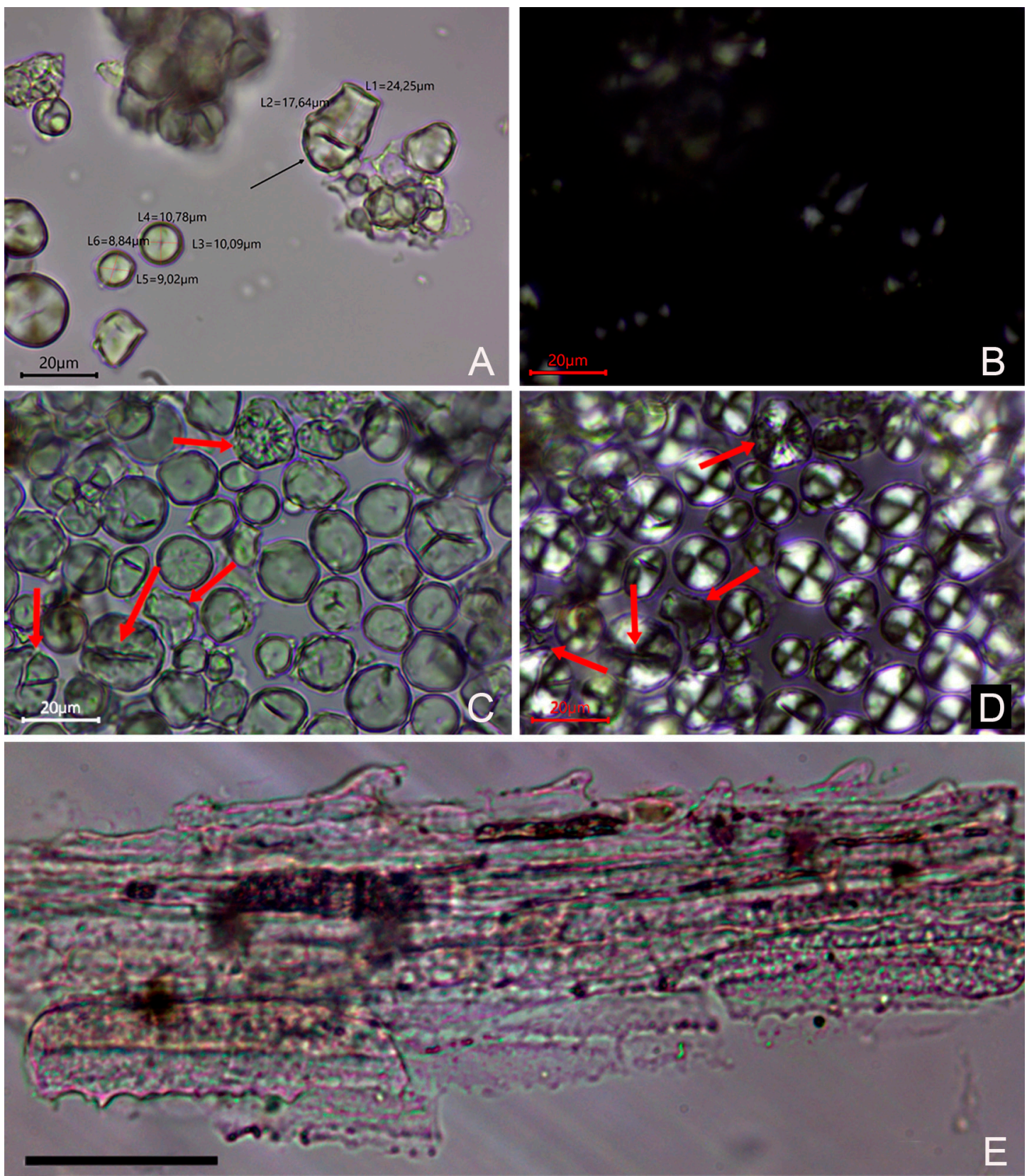

Fig. 3. Micrografías de la colección de referencia de fitolitos y granos de almidón. A-D) Granos de almidón de Zea mays L. registrados en una muestra de "harina para api". A) Registro morfométrico de los granos de almidón, la flecha señala un grano poliédrico. B) Mismos granos vistos con luz polarizada. C) Registro de los daños tafonómicos producidos por las prácticas culinarias. Las flechas rojas señalan daños en las fisuras e hilos y fracturas. D) Misma imagen con luz polarizada. E) Morfotipos de fitolitos articulados de calcinados de Poaceae. Escala: $20 \mu \mathrm{m}$.

Fig. 3. Reference collection micrographs of phytoliths and starch grains. A-D) Zea mays L. starch grains registered in a "flour for api" sample: A) Starch grains morphometric record, the arrow indicates a polyhedral grain. B) The same grains seen with polarized light. C) Record of the taphonomic damage produced by culinary practices. Red arrows indicate damage to the striae and hilum and fractures. D) Same image with polarized light. E) Morphotypes of articulated phytoliths of calcined Poaceae sample. Scale: $20 \mu \mathrm{m}$. 
el estándar Darwin Core ( $\mathrm{DwC})$. Los campos aplicados en nuestra base de datos son: ID del Registro biológico (occurrenceID), base del Registro (basisOfRecord), código de la institución (institutionCode), código de la Colección (collectionCode), número de catálogo (catalogNumber), otro número de catálogo (otherCatalogNumber), clasificación superior (higherClassification), reino (kingdom), filo (phylum), clase (class), orden (order), familia (family), género (genus), epíteto específico (specificEpithet), epíteto intraespecífico (infraspecificEpithet), autoría del nombre científico (scientificNameAuthorship), calificador de la identificación (identificationQualifier), nombre científico (scientificName), categoría del taxón (taxonRank), estado nomenclatural (nomenclaturalCode), nombre vernáculo (vernacularName), geografía superior (higherGeography), continente (continent), país (country), código del país (countryCode), departamento (stateProvince), municipio (county), cabecera municipal / centro poblado (municipality), localidad (locality), localidad original (verbatimLocality), longitud decimal (decimalLongitude), latitud decimal (decimalLatitude), longitud original (verbatimLongitude), latitud original (verbatimLatitude), datum geodésico (geodeticDatum), incertidumbre de las coordenadas en metros (coordinateUncertaintyInMeters), elevación original (verbatimElevation), elevación mínima en metros (minimumElevationInMeters), elevación máxima en metros (maximumElevationInMeters), fecha del evento (eventDate), citación bibliográfica (bibliographicCitation), registrado por (recordedBy), número del registro (recordNumber) y preparaciones (preparation). Además, se incluyen campos sobre atributos cualitativos y cuantitativos con los caracteres de cada tipo de parte útil de la planta (semillas, frutos, hojas, flores, raíces o fibras). En caso de estar disponible, se detalla información sobre usos y tipos de procesamientos culinarios (recetas).

$\mathrm{DwC}$ facilita el intercambio de información sobre la diversidad biológica proporcionando identificadores, campos predeterminados que pueden ser controlados o no y definiciones. Permite la posterior publicación de estos datos primarios en portales de datos, como el de la Global Biodiversity Information Facility - GBIF (www.gbif. org) (Wieczorek et al., 2012; Darwin Core Maintenance Group, 2021).

1.3.2. Bloque II. Granos de almidón.- Los granos de almidón son polímeros de carbohidratos que sirven como forma principal de almacenamiento de la energía de las plantas. Los polímeros orgánicos que los componen se denominan amilosa y amilopectina (Esau, 1976). Los atributos utilizados para su descripción y clasificación varían considerablemente entre las especies, géneros y familias, permitiendo su distinción y agrupamiento a nivel taxonómico. Dichos atributos fueron seleccionados de acuerdo a las provisiones del International Code for Starch Nomenclature (ICSN, 2011) y criterios adicionales proporcionados por Babot (2007) y Pagan Jiménez (2015).

Para describir la morfología básica de un grano de almidón se tomó en cuenta, su forma en términos bidimensionales y tridimensionales. Los términos bidimensionales se describieron en la vista en planta y en el perfil. Las medidas del largo, ancho y/o diámetro, proveyeron información relevante para diferenciar géneros, o especies de un mismo género. También se tuvo en cuenta el hilo o hilum, punto de inicio de crecimiento de los gránulos. Se indicó la visibilidad o no del laminado, el 
cual está ligado al patrón de crecimiento de las moléculas de amilosa y amilopectina de los gránulos. Las lamelas no son visibles en todos los taxa. También se describió la presencia y tipos de fisuras, las cuales se ubican mayormente en donde se encuentra el hilo cuando se trata de atributos naturales del grano. En ciertos especímenes, las fisuras son marcadamente homogéneas, pero en otros pueden presentar relativa variabilidad de tipos dentro de un mismo taxón. Al describir los bordes de los gránulos se registraron las diferencias en las líneas externas que los delimitan, principalmente entre especies de un mismo género o razas y variedades dentro de una misma especie (Pagán Jiménez, 2015).

Por último, entre las características morfológicas, se registró el aspecto de la cruz de extinción. Los almidones, al ser cuerpos semicristalinos y con estructura molecular ordenada, cuando son observados con microscopio de polarización proyectan una cruz negra sobre el cuerpo de color blanco brillante. La mayoría de los gránulos de almidón cuentan con estas propiedades de polarización generando cruces de extinción que, en ocasiones, son características o representativas del género o especie del órgano vegetal de origen (ICSN, 2011; Babot, 2007 y Pagan Jiménez, 2015).

Los últimos atributos consignados en esta parte de la base de datos se refieren a la multiplicidad de los granos presentes en el taxón, y las ilustraciones y micrografías que complementan los datos indicados en los párrafos precedentes.

1.3.3. Bloque III. Fitolitos.- Los fitolitos son biominerales que se forman en los tejidos vegetales. Cuando los tejidos que los contienen son degradados, pasan a incorporarse a los suelos, en donde pueden preservarse miles de años. Son producidos por muchas familias de plantas, en algunas con significado taxonómico, porque mantienen las características morfológicas que son dependientes de su génesis. Las especies herbáceas producen mayor cantidad de células silicificadas que las arbóreas, siendo las de las familias Poaceae, Cyperaceae y Arecaceae las máximas productoras, incluso con morfotipos diagnósticos (Bertoldi de Pomar, 1975; Piperno y Pearsall, 1998; Piperno, 2006). En la caracterización del conjunto de fitolitos de un taxón, es preciso considerar a los morfotipos que son típicos en tales conjuntos, sean estos diagnósticos o solo característicos. Así se obtiene una visión más completa de la producción de fitolitos de cada taxón (Babot et al., 2017). Para el registro de la frecuencia relativa de los morfotipos se utilizaron los atributos definidos en el ICPN 2.0 (ICPT, 2019), los cuales son mencionados a continuación.

Lo primero que debe indicarse es el origen anatómico y la ocurrencia taxonómica de cada morfotipo fitolítico. Luego, se indican los diferentes nombres propuestos por el código. A saber, el nombre taxonómico, el anatómico y el morfológico. Este último se define a partir de la forma general del fitolito y su textura y/u ornamentación. En relación a los atributos cuantitativos de tamaños, se midió el largo y ancho en micrones, de acuerdo a criterios morfométricos. Luego, se describieron y caracterizaron consignando la forma 2-D y 3-D, los grados, planos y ejes de simetría, la textura de la superficie y, en caso de presentar, su ornamentación. Por último, se indicó si el morfotipo presenta inclusiones. 
Para ilustrar las descripciones se realizaron dibujos lineales y se tomaron micrografías con la cámara del microscopio. En algunos casos particulares, como los continuos morfológicos (Musaubach et al., 2019; Babot et al., 2016 y 2017) se incluyeron imágenes 3D diseñadas con el programa de diseño 3D, Z Brush (c) 2015 Pixologic, Inc., así como micrografías tomadas con microscopio electrónico de barrido (MEB).

\section{CONSIDERACIONES FINALES}

La propuesta de conformación de una base de datos digital del muestrario y colección de referencia de granos de almidón y fitolitos, así como la incorporación de nuevos ejemplares al Herbario JUA, vinculados al patrimonio culinario de los Andes Centro Sur, se inserta en la tendencia global a la accesibilidad de la información. La misma se encuentra en construcción y se propone contribuir en el mediano plazo al acceso abierto del conocimiento producido sobre el tema, siguiendo los criterios FAIR. Este tipo de esquema sólo recientemente ha comenzado a ser aplicado en relación al conocimiento arqueobotánico por lo que se trata de una iniciativa piloto. Además de adecuar nuestras colecciones a los requerimientos de la ciencia moderna, estos esquemas benefician la propia práctica disciplinaria. Mediante la sistematización de la colección y del material de referencia se logran mayores precisiones en la identificación arqueobotánica de restos arqueológicos de preparaciones basadas en plantas andinas, así como de las prácticas de elaboración vinculadas. Además de la utilidad de este material de referencia para el estudio arqueobotánico, su descripción y registro permite no solo su catalogación como parte del patrimonio del Herbario JUA, sino que también aporta a la valorización del patrimonio biocultural comunitario jujeño. Dichos conocimientos podrán ser utilizados como insumo para propiciar o reforzar procesos de puesta en valor del patrimonio cultural de la Provincia de Jujuy, agregado de valor al mismo, su visibilización y difusión a diferentes escalas (comunitaria, local, regional y extra-regional mediada por el turismo). $\mathrm{Al}$ recuperar y valorizar estos productos culinarios, saberes y prácticas tradicionales, podrán ser integrados al acervo patrimonial referido a las formas ancestrales de preparación, servicio y consumo de los cultivos andinos, incrementando de esta manera nuestro capital patrimonial cultural y gastronómico tangible como intangible. Finalmente, mediante esta colección y el acceso abierto de los datos primarios de la base de datos, se contribuye a la valorización, visibilización, salvaguardia y gestión del patrimonio culinario quebradeño.

\section{AGRADECIMIENTOS}

Esta investigación se financió con el proyecto SECTER "Estudio arqueobotánico del patrimonio culinario prehispánico Surandino y sus prácticas asociadas. La Quebrada de Humahuaca como caso de estudio" (Desafíos en la Investigación UNJu) DU-08; y los proyectos PICT 2017-0280 y PICT 2018-0584. Agradecemos a los revisores anónimos, por la predisposición y velocidad para la evaluación del manuscrito. 


\section{BIBLIOGRAFÍA}

Albeck, M. (1992/1993). Áreas agrícolas y densidad de ocupación prehispánica en la Quebrada de Humahuaca. Avances en Arqueología 2: 56-77.

Arenas, P. y Martínez, G. J. (2012). Estudio Etnobotánico en Regiones Áridas y Semiáridas de Argentina y Zonas Limítrofes: Experiencias y reflexiones metodológicas de un grupo de investigación. En: P, Arenas, Kamienkowski, N. (Eds.), Etnobotánica en zonas áridas y semiáridas del Cono Sur de Sudamérica (pp. 11-43). Buenos Aires: Consejo Nacional Investigaciones Científicas Técnicas (CONICET).

Babot, P. (2007). Granos de almidón en contextos arqueológicos: posibilidades y perspectivas a partir de casos del Noroeste argentino. En: B. Marconetto, Babot, P., Oliszewski, N. (Comps.), Paleoetnobotánica del Cono Sur: estudios de casos y propuestas metodológicas, (pp. 95-125). Córdoba: Museo de Antropología, Facultad de Filosofía y Humanidades, Universidad Nacional de Córdoba.

Babot, P. y Musaubach, M. G. (2019). Silicofitolitos como herramientas para el estudio arqueológico de la colecta y post-colecta de gramíneas silvestres y cereales en los Andes Centro Sur. Resúmenes de las XXXVII Jornadas de la Sociedad Argentina de Botánica, Boletín de la Sociedad Argentina de Botánica 54 (Suplemento): 28-29.

Babot, P., Lund, J. y Olmos, A. V. (2014). Taphonomy in the kitchen: culinary practices and processing residues of native tuberous plants of the South-Central Andes. Intersecciones en Antropología, Special Issue $\mathrm{N}^{\circ} 1$ Taphonomic Approaches to the Archaeological Record: $35-53$.

Babot, M. P., Musaubach, M. G. y Gonzalez, J. A. (2016). Continuo morfológico y fitolitos 3D. Aportes desde una perspectiva arqueobotánica para definir conjuntos fitolíticos. En Zucol, A. F., Patterer, N. I., Colobig, M. M. \& Moya, E. (eds.). Taller de Micropaleoetnobotánica. Relevancia de una Red Interdisciplinaria de Investigaciones en Fitolitos y Almidones, Libro de Resúmenes, 79.

Babot, M. P., Musaubach, M. G. y Plos, A. (2017). An archaeobotanical perspective in the study of inflorescence phytoliths of wild grasses from arid and semiarid environments of Argentina. Quaternary International 434: 129-141. DOI: 10.1016/j.quaint.2016.02.045.

Bertoldi de Pomar, H. (1975). Los silicofitolitos: sinopsis de su conocimiento. $D a$ rwiniana 19 (24):173-206.

Crowther, A. (2012). The differential survival of native starch during cooking and implications for archaeological analyses: a review. Archaeological and Anthropological Sciences 4: 221-235.

Darwin Core Maintenance Group. (2021). List of Darwin Core terms. Biodiversity Information Standards (TDWG). http://rs.tdwg.org/dwc/doc/list/

Dickau, R. (2005). Resource use crop dispersals, and the transition to agriculture in prehistoric Panama: evidence from starch grains and macroremains. Doctoral Dissertation, Temple University. Philadelphia.

Esau, K. (1976). Anatomía Vegetal. Barcelona: Ed. Omega. 
ICSN (2011). The International Code for Starch Nomenclature, www.fossilfarm. org/ICSN/Code.html

ICPT (2019). International Committee for Phytolith Taxonomy - International Code for Phytolith Nomenclature (ICPN) 2.0, Annals of Botany 124 (2): 189199. https://doi.org/10.1093/aob/mcz064

Johansen, D. A. (1940). Plant microtechnique. New York: McGraw-Hill Book Co.

Kansa, S. W., Atici, L., Kansa, E. C. y Meadows, R. H. (2020). Archaeological analysis in the information age: guidelines for maximising the reach, comprehensiveness and longevity of data. Advances in Archaeological Practice 8 (1): 40-52. https://doi.org/10.1017/aap.2019.36

Karoune, E. (2020). Data from "Assessing Open Science Practices in Phytolith Research". Fournal of Open Archaeology Data 8: 6. https://doi.org/10.5334/joad.67

Katinas, L. (2001). El Herbario: Significado, Valor y Uso. Serie Técnica y Didáctica Número 1. La Plata: PROBIOTA.

Korstanje, M. A. y Babot, P. (2007). Microfossils Characterization from South Andean Economic Plants. En: M. Madella, Zurro, D., (Eds.), Plant, People and Places: Recent Studies in Phytolith Analysis, pp. 41-72. Cambridge: Oxbow Books.

Lambaré, D. A., Vignale, Nilda Dora y Pochettino, M. L. (2015). Las ferias y festivales regionales como instancia de reafirmación del patrimonio biocultural en la quebrada de Humahuaca (Jujuy, Argentina): El intercambio de duraznos (Prunus persica). Gaia Scientia 9 (12): 1-9.

Lodwick, L. (2019). Sowing the seeds of future research: data sharing, citation and reuse in Archaeobotany. Open Quaternary 5 (7): 1-15. https://doi.org/10.5334/oq.62

López Geronazzo, L., Musaubach, M. G. y Otero, C. (2019). Explorando protocolos interdisciplinarios para el estudio de plantas y animales en ajuares funerarios quebradeños. Presentado en XIII Jornadas Regionales de Investigación en $\mathrm{Hu}-$ manidades y Ciencias Sociales. Facultad de Humanidades y Ciencias Sociales. San Salvador de Jujuy. Argentina.

Musaubach, M. G. y Babot, P. (2019). Elementos para explorar el uso de gramíneas silvestres de ambientes áridos de los Andes Centro Sur: primeras aproximaciones desde los conjuntos fitolíticos de inflorescencias e infrutescencias. Revista del Museo de Antropología de Córdoba 12 (1): 55-70.

Musaubach, M. G. y Scaro, A. (2019). Primeras aproximaciones al estudio arqueobotánico de contenidos vegetales en tártaro dental de individuos de Esquina de Huajra (Quebrada de Humahuaca, Jujuy). En: E. Alfaro Gómez, Revollo, G. B. y Cháves, E. R. Libro de Resúmenes de las Decimocuartas Jornadas Nacionales de Antropología Biológica. (Comp.), p. 83.

Musaubach, M. G. y Scaro, A. (2020a). Identifying Andean crop processing and consumption in the area of Quebrada de Humahuaca (Argentina) under Inca domination. 26th EAA-V. A. M. - Abstract Book, p. 57. Praga: European Association of Archaeologists. www.e-a-a.org/eaa2020virtual

Musaubach, M. G. y Scaro, A. (2020b). Mote: an ancient recipe in Andean kitchens. Experimental and taphonomic analysis. 26th EAA Virtual Annual Meeting Abstract Book. Praga: European Association of Archaeologists, pp. 57-58. www. e-a-a.org/eaa2020virtual 
Musaubach, M. G., Plos, A. y Babot, M. P. (2013). Differentiation of Maize (Zea mays L.) from native wild grasses based on starch grain morphology. An Archaeobotanical perspective from Central Pampas of Argentina. Fournal of Archaeological Science 40 (2): 1186-1193.

Musaubach, M. G., Scaro, A. y Heit, C. (2019). Estudio de las Raíces Prehispánicas del Patrimonio Gastronómico de la Quebrada de Humahuaca. Presentado en II Jornadas Etnobiología y Sociedad. Diversidad de Actores, Múltiples Naturalezas. Universidad Nacional de Córdoba. Córdoba. Argentina.

Nielsen, A. (2010). Celebrando con los antepasados, Arqueología del Espacio Público en Los Amarillos, Quebrada de Humahuaca, Fujuy, Argentina. Buenos Aires: Mallku Ediciones.

Ortiz, G. y Heit, C. (2012). Consumo y economía en los grupos pedemontanos tempranos de la cuenca del San Francisco (Jujuy, Argentina). En: P. Babot, M. Marschoff y F. Pazzarelli (eds.) Las manos en la masa: arqueologías, antropologías e historias de la alimentación en Suramérica, pp. 621-638. Córdoba: FFyH, UNC.

Pagán Jiménez, J. R. (2015). Almidones. Guía de material comparativo moderno del Ecuador para los estudios paleoetnobotánicos en el Neotrópico. Volumen I. Buenos Aires: Aspha Ediciones.

Pazzarelli, F. (2008). Notas acerca de una arqueología de la comida. La Zaranda de Ideas. Revista de fóvenes Investigadores en Arqueología 4: 157-162.

Pazzarelli, F. y Lema, V. (2018). A Pot Where Many Worlds Fit: Culinary Relations in the Andes of Northern Argentina. INDIANA 35 (2): 271-296.

Peto, Á., Gyulai, F., Pópity, D. y Kenéz, Á. (2013). Macro- and micro-archaeobotanical study of a vessel content from a Late Neolithic structured deposition from southeastern Hungary. Fournal of Archaeological Science 40: 58-71.

Piperno, D. (2006). Phytoliths. A Comprehensive Guide for Archaeologist and Paleoecologist. Altamira Press.

Piperno, D. R. y Pearsall, D. M. (1998). The Silica Bodies of Tropical American Grasses: Morphology, Taxonomy, and Implications for Grass Systematics and Fossil Phytolith Identification. Smithsonian Contributions to Botany. 1-40. https://doi. org/10.5479/si.0081024X.85

Raviele, M. (2011). Experimental assessment of maize phytolith and starch taphonomy in carbonized cooking residues. Fournal of Archaeological Science 38 (10): 2708-2713.

Vignale, N. D. y Gurni, A. A. (2001). Diferenciación de especies equisetiformes utilizadas en medicina tradicional en la provincia de Jujuy, Argentina. Dominguezia 17 (1): 23-30.

Villalba, M. S. (2019). Etnovariedades de Malus domestica (Rosaceae) en la comunidad de Ocumazo (Humahuaca): etnobotánica y criterios de clasificación local. Tesis de Grado, Universidad Nacional de Jujuy, Argentina.

Wieczorek J., Bloom, D., Guralnick, R., Blum, S., Döring, M. Robertson, T. y Vieglais, D. (2012). Darwin Core: An Evolving Community-Developed Biodiversity Data Standard. PLoS ONE 7 (1): e29715. https://doi.org/10.1371/journal. pone.0029715 
Wilkinson, M. D., Dumontier, M., Aalbersberg, L. J., Appleton, G., Axton, M., Baak, A., Blomberg, N., Boiten, J.-W., da Silva Santos, L. B., Bourne, P. E., Bouwman, J., Brookes, A. J., Clark, T., Crosas, M., Dillo, I., Dumon, O., Edmunds, S., Evelo, C. T., Finkers, R., Gonzalez-Beltran, A., Gray, A. J. G., Groth, P., Goble, C., Grethe, J. S., Heringa, J., 't Hoen, P. A. C, Hooft, R., Kuhn, T., Kok, R., Kok, J., Lusher, S. J., Martone, M. E., Mons, A., Packer, A. L., Persson, B., Rocca-Serra, P., Roos, M., van Schaik, R., Sansone, S.A., Schultes, E., Sengstag, T., Slater, T., Strawn, G., Swertz, M. A., Thompson, M., van der Lei, J., van Mulligen, E., Velterop, J., Waagmeester, A., Wittenburg, P., Wolstencroft, K., Zhao, J. y Mons, B. (2016). The FAIR Guiding Principles for scientific data management and stewardship. Scientific Data, 3, 160018. http://dx.doi. org/10.1038/sdata.2016.18

Woelfle, M., Olliaro, P. y Todd, M. H. (2011). Open science is a research accelerator. Nature Chemistry 3 (10): 745-748. DOI:10.1038/nchem.1149

Zarlavsky, G. E. (2014). Histología vegetal. Técnicas simples y complejas. Buenos Aires: Sociedad Argentina de Botánica. Buenos Aires, Argentina. 